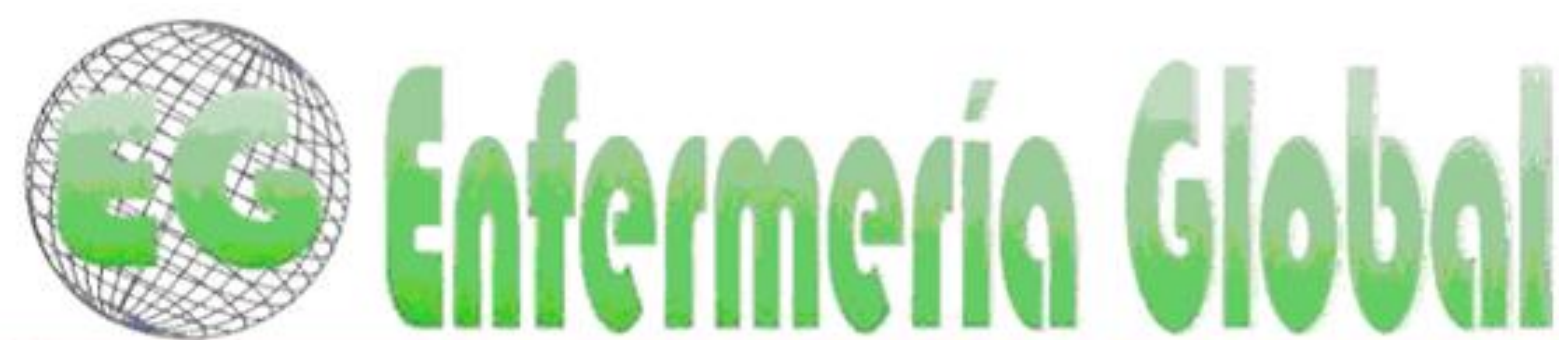

ISSN 1695-6141 $N^{\circ} 43$

\title{
Barreras para la utilización de la investigación. Estudio descriptivo en profesionales de Enfermería en un hospital del sudoeste de Madrid
}

Barriers to the utilisation of research. Descriptive study performed on nurses at a hospital in the southwest of Madrid

\section{${ }^{*}$ Guadarrama Ortega, David} *Enfermero. Unidad de Investigación. Hospital Universitario Fundación Alcorcón. Madrid. España. E-
mail: $\underline{\text { dguadarrama@fhalcorcon.es }}$

Palabras clave: Enfermería; Barreras; Utilización de la investigación.

Keywords: Nursing; Nurses; Barriers; Research Utilization

\section{RESUMEN}

Objetivos. Identificar las situaciones, circunstancias o barreras que dificultan a la enfermería utilizar los resultados de las investigaciones en el Hospital Universitario Fundación Alcorcón (HUFA), así como sus facilitadores.

Objetivos específicos. Evaluar si existen diferencias en la puntuación de la escala THE BARRIERS en función de las distintas variables estudiadas.

Métodos. Estudio observacional, descriptivo y transversal durante mayo 2014. La población de estudio fueron todas las enfermeras que trabajan en el HUFA. El cuestionario que incluye la escala THE BARRIERS de S.Funk et al. se facilitó mediante una encuesta on-line distribuida a través del correo corporativo del hospital.

Resultados. Respondieron 69 enfermeras (16\%), fueron válidas 67 encuestas. Las barreras identificadas con mayor puntuación fueron: "La enfermera desconoce las investigaciones" media=3.33 ( $D E=0.89) ; y$ "Los médicos no colaboran en la implementación" media=3.26 (DE=0.86). Se encuentran diferencias estadísticamente significativas en la suma de la escala THE BARRIERS en función de la experiencia como enfermera, la formación post-grado, y haber realizado investigaciones previas. No se encuentran diferencias significativas en función del resto de variables.

Conclusiones. Aunque la falta de tiempo se identifica como la principal barrera para la investigación en la mayoría de los estudios, aquí aparece por detrás de otras como el desconocimiento de las investigaciones, la falta de colaboración, o que la información no está recogida en un solo lugar. Sin embargo el tiempo aparece como una de las principales barreras en las respuestas de texto libre. 


\section{ABSTRACT}

Objectives: To identify barriers to the utilization of research as perceived by nurses at the University Hospital Alcorcón (HUFA) Foundation and their facilitators.

Specific objectives: To assess the differences in scale scores THE BARRIERS depending on the different variables studied.

Methods: A cross sectional study was conducted during May 2014. The study population were all nurses working in the HUFA. The questionnaire included THE BARRIERS scale of S.Funk et al. It was facilitated by an online survey distributed through the corporate mail hospital.

Results: 69 nurses (16\%) completed the survey, 67 surveys were valid. The barriers identified with the highest score were: "The nurse is unaware of the research" mean $=3.33(\mathrm{SD}=0.89)$; and "Physicians will not cooperate with implementation" mean $=3.26(S D=0.86)$. Statistically significant differences were found in the total score of THE BARRIERS scale based on experience as a nurse, post-graduate training, and have made previous research. No significant differences were found in terms of other variables.

Conclusions: Although lack of time is identified as the main barrier to research in most of the studies, here appears behind other as the unknowing of research or lack of cooperation. But lack of time appears as one of the main barriers in free text responses.

\section{INTRODUCCIÓN}

\section{Antecedentes}

Tradicionalmente, la enfermería no se ha basado en las evidencias científicas, y su práctica se ha sustentado en la tradición y la experiencia. A su vez, las enfermeras no han recibido formación en investigación. No obstante, tampoco hay una cultura favorable a la investigación en muchos profesionales de enfermería ${ }^{(1)}$. Sin embargo, el paso que en 1977 dio la profesión enfermera en España accediendo al ámbito de la universidad $^{(2)}$, ha supuesto de forma creciente el desarrollo de investigaciones realizadas por enfermeros ${ }^{(3)}$. La enfermería española comenzó a realizar investigaciones en forma de tesis doctorales ${ }^{(3)}$, y esta producción científica de temática enfermera ha aumentado progresiva y exponencialmente ${ }^{(3)}$.

Desde los años 90 del siglo XX se viene investigando en los motivos o barreras que las enfermeras identifican como aquellas que impiden la investigación y su utilización posterior en su práctica clínica ${ }^{(4-6)}$. En 1991 Sandra G. Funk plantea la Escala THE BARRIERS en la que se medían aquellas circunstancias que, según las enfermeras, dificultan la utilización de la investigación disponible ${ }^{(4)}$.

S. Funk clasificó las distintas "barreras" en cuatro grupos o "Factores": Características del profesional, características de la organización, calidad de la investigación, y presentación-accesibilidad de ésta ${ }^{(4)}$. En la misma línea, Hunt encuentra distintos factores que han llevado a las enfermeras a estar alejadas de la investigación y de la evidencia, ya que muchas no los comprenden, otras no los creen, otro grupo no sabe cómo hacer uso de ellos, incluso, a algunas no les está permitido aplicar hallazgos procedentes de la investigación ${ }^{(6)}$. Hunt sugiere que las barreras a la utilización de la investigación abarcan cinco dominios: la calidad de la investigación, el acceso a la investigación,el proceso de utilización de la investigación, la actitud y el conocimiento de enfermeras, y la organización en la que los resultados de investigación se van a aplicar $^{(6)}$. 
En 2010 se publicó un estudio en el cual se describían las barreras para la utilización de la investigación en centros de toda España ${ }^{(7)}$, y que servirá como referencia para comparar con los resultados de nuestro estudio. En el citado estudio las 3 barreras identificadas con mayor puntuación son: "No hay tiempo suficiente en el trabajo para implementar nuevas ideas", seguida de "La enfermería desconoce las investigaciones" y de "Los médicos no colaboran en la implementación"(7).

\section{Estado actual del tema.}

La enfermería investiga poco en relación a otras disciplinas científicas. Por poner un ejemplo, en una búsqueda rápida en PubMed utilizando los descriptores "Nursing" y "Medicine" obtenemos algo más de 219.000 resultados en Enfermería (Fig.1) frente a los más de 800.000 artículos de Medicina. De hecho, en esta base de datos, existe el descriptor "Nursing Research" mientras que el equivalente en medicina no aparece, dando por supuesto que la medicina lleva asociada consigo la investigación. También podemos observar cómo los estudios incluidos en el descriptor "Nursing" han crecido exponencialmente. (Fig.1) Si bien es cierto que la Enfermería española se incorporó relativamente tarde a la Universidad (ámbito científico por excelencia) desde su paso a titulación universitaria ha venido aumentando exponencialmente su actividad científica en forma de artículos publicados. (Fig.2) Y aunque se viene haciendo hincapié en su importancia por parte de las escuelas de Enfermería, sigue siendo una de las profesiones que menos investiga del ámbito sanitario (no olvidemos que las enfermeras son el estamento más numeroso dentro de este ámbito).

Tampoco podemos ignorar que la enfermería suele mayoritariamente difundir los resultados de su investigación en congresos y jornadas frente a la publicación en revistas científicas.

Fig. 1 Evolución del número de artículos incluidos en el descriptor "Nursing" en el tesauro "Mesh" hasta el año 2013

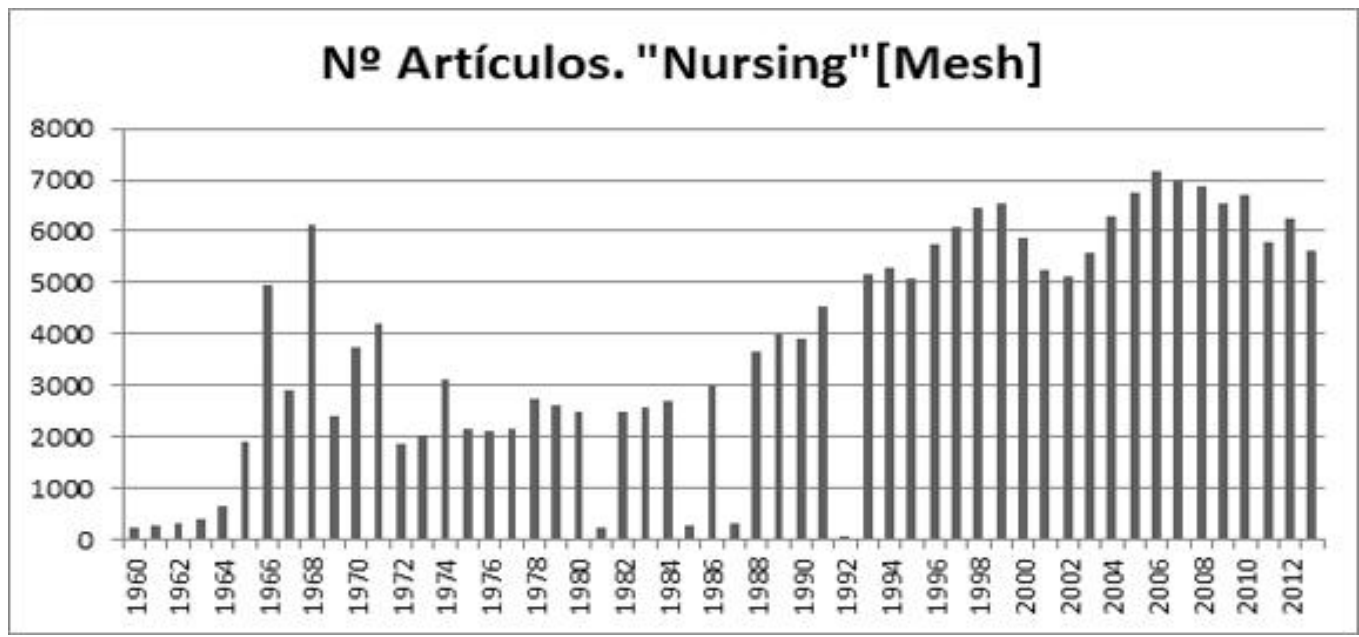


Fig. 2 Evolución del número de artículos publicados con la búsqueda "Nursing" AND "Spain" en la Web Of Science.

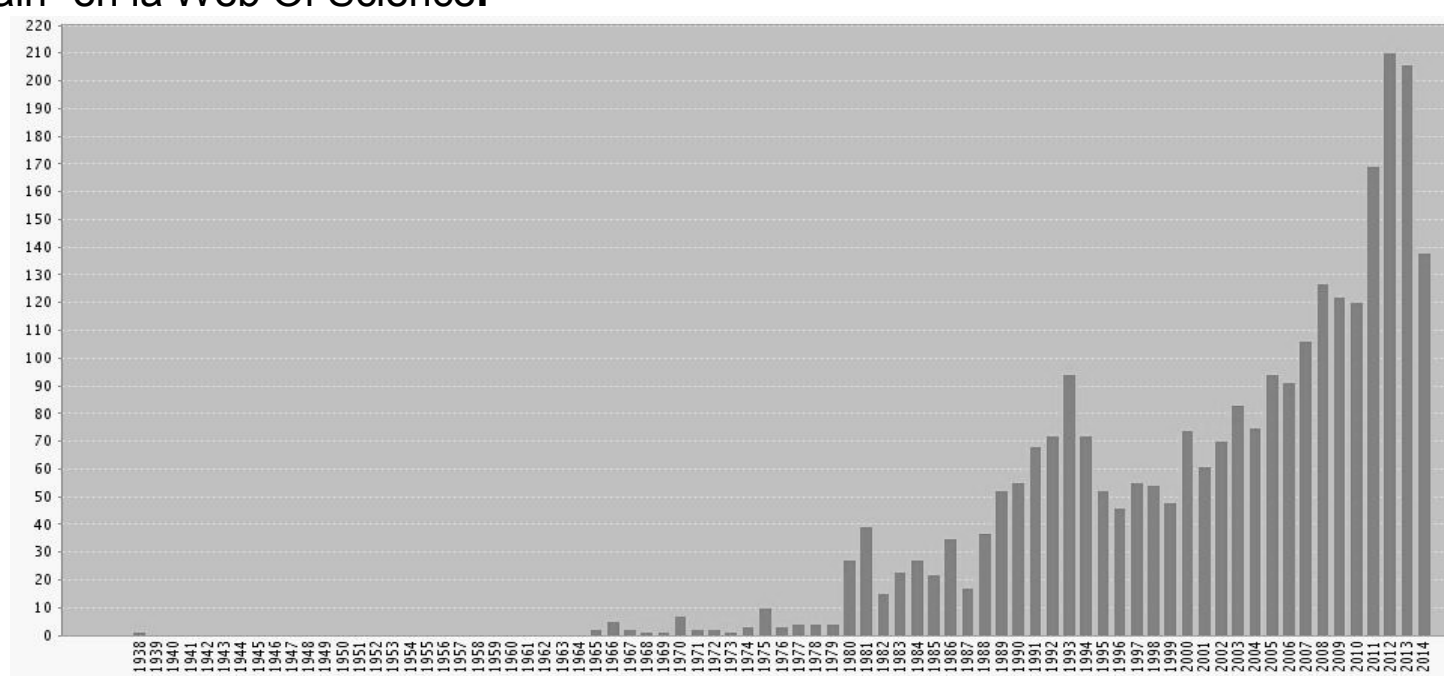

\section{Justificación.}

Desde la apertura en 1998 del Hospital Universitario Fundación Alcorcón, la enfermería del centro ha realizado una labor investigadora potente sobre todo teniendo en cuenta el número de profesionales de enfermería del mismo si lo comparamos frente a otros hospitales de mayor tamaño de la Comunidad de Madrid.

Sin embargo, muchos profesionales de enfermería manifiestan sus dificultades para investigar y posteriormente utilizar esos resultados de investigación. Es a raíz de estas reflexiones de las que parte la necesidad de estudiar este fenómeno en nuestro centro.

El estudio que se pretende realizar pretende conocer y dar respuesta a las necesidades y dificultades que el personal de Enfermería del H.U. Fundación Alcorcón tiene en relación a la aplicación de cuidados basados en la evidencia científica.

\section{OBJETIVOS}

El objetivo principal del estudio es identificar las principales barreras que dificultan a la enfermería utilizar los resultados de las investigaciones en el Hospital Universitario Fundación Alcorcón, así como sus facilitadores mediante el uso de la escala THE BARRIERS de S.Funk.

Objetivos específicos:

- Evaluar si existen diferencias en la puntuación de la escala THE BARRIERS en función de la edad y sexo, los años de experiencia, formación postgrado, la unidad en la que se trabaja, las responsabilidades familiares, formación en investigación y experiencia en investigación. 


\section{MATERIAL Y MÉTODOS}

\section{Diseño}

Se realiza un estudio observacional, descriptivo y transversal, que se realizó durante el año 2014 en el Hospital Universitario Fundación Alcorcón. Madrid.

Se realizó una búsqueda bibliográfica que permitiera contextualizar la investigación. Para ello se utilizarán diferentes bases de datos o fuentes documentales: CINAHL, PubMed, CUIDEN, entre otras. Las palabras clave para la búsqueda bibliográfica serán: BARRERAS, INVESTIGACIÓN EN ENFERMERÍA (DESCRIPTOR). NURSING RESEARCH, BARRIERS.

\section{Población de estudio}

La población de estudio fueron todas las enfermeras que trabajan en el H.U. Fundación Alcorcón, ya sean estas asistenciales o gestoras.

Los criterios de inclusión fueron: Enfermeras del H.U. Fundación Alcorcón que disponen de dirección de correo electrónico activo en el momento de la distribución de la encuesta.

Debido al número de enfermeras que constan en la última memoria del hospital (440 incluyendo residentes de las especialidades) se decide incluir a todos los profesionales de enfermería en el tamaño muestral ya que es fácilmente manejable y la distribución de la encuesta se realizará vía e-mail.

\section{Recogida de datos}

El cuestionario con la escala THE BARRIERS se facilitó mediante una encuesta online distribuida a través del correo corporativo del hospital en varios momentos para facilitar su cumplimentación. Para garantizar confidencialidad se utilizó el buzón de correo electrónico "HUFA Enfermeras" al cual se remitió la encuesta vía e-mail. El cuestionario se alojó en una plataforma web para la realización de encuestas que permite la explotación de los datos posteriormente, en este caso se elige Google Docs ${ }^{\circledR}$ en concreto su opción de formularios.

\section{Análisis de datos}

Se realizó un análisis descriptivo de todas las variables, aportando los resultados en forma de porcentajes (cualitativas) y media y desviación típica (cuantitativas). Las variables recogidas en el cuestionario fueron:

- Edad: variable cuantitativa medida en años cumplidos

- Sexo: variable cualitativa dicotómica (1=hombre; $2=$ mujer)

- Años de experiencia: variable. cuantitativa medida en años cumplidos.

- Formación Postgrado: variable cualitativa nominal.

- Unidad: variable cualitativa nominal.

- Cargas familiares: variable cualitativa nominal.

- Trabajos previos de investigación: variable cualitativa dicotómica (1=Si; 2=No)

- Frecuencia de lectura de artículos científicos: variable cualitativa nominal. 
- Formación en temas de investigación en los últimos tres años: variable cualitativa dicotómica $(1=\mathrm{Si} ; 2=\mathrm{No})$

Para facilitar el análisis de los datos, debido a la escasa muestra, algunas de estas variables han sido transformadas en cualitativas dicotómicas (Años de experiencia, edad, cargas familiares, formación Post-grado,

- Escala THE BARRIERS. La escala THE BARRIERS, publicada por S.Funk et al. en 1991, está compuesta por 29 ítems clasificados en 4 factores. Cada ítem se puntúa del 1 al 4 (1. Nunca, 2. En menor grado, 3. En grado moderado, 4. En gran parte), existiendo una quinta opción que no tiene asignada ningún valor y que corresponde con la opción "No tengo opinión". Los ítems fueron derivados de la literatura, a partir de datos de investigación, y desde el Proyecto CURN (CURN Project Research Utilization Questionnaire (Crane, Pelz, \& Horsley, 1977).

Los cuatro factores en los que se clasifican los ítems son: características del profesional, características de la organización, características de los resultados o calidad de la investigación, y características relacionadas con la accesibilidad de los resultados.

Hay que tener en cuenta que el ítem número 27 no está clasificado en ninguno de los factores, sin embargo se ha mantenido en la escala debido a los comentarios de profesionales dado su interés. La suma máxima de los ítems de cada factor es 32 para los dos primeros y 24 para los dos últimos.

Hay dos versiones de la escala a disposición de los investigadores:

- La escala THE BARRIERS original, descrita en Applied Nursing Research en 1991, que pide a los encuestados que proporcionen sus percepciones generales de las barreras para la utilización de la investigación.

- Y una segunda versión que personaliza las instrucciones de modo que las respuestas se proporcionan en relación con el propio lugar de trabajo del individuo.

Para nuestro estudio se ha utilizado la segunda versión debido a que se pretendía obtener los resultados únicamente de nuestro centro.

El análisis de los datos se realizó con el programa de análisis estadístico SPSS y Excel. Para los contrastes de hipótesis se utilizaron los test de correlación de Pearson y $\mathrm{T}$ de Student en función del tipo de variable de estudio. También, para determinar el nivel de significación estadística se estableció una $p<0.05$.

\section{RESULTADOS}

Respondieron a la encuesta 69 de las 417 enfermeras que conformaban la plantilla del Hospital Universitario Fundación Alcorcón. Se consideraron válidas 67 de las 69 encuestas recibidas. 


\section{Análisis de las variables socio-demográficas y relacionadas con la profesión}

- La edad media de la muestra es 38,75. (DE: 6.8) en un rango de de 22 a 55 años.

- El 83,6\% de las enfermeras eran mujeres, frente al 16,4 de hombres.

- La media de años de experiencia como enfermera era de 16,73 (DE: 6,3) Rango de 1 a 34 años.

- En cuanto a la formación post-grado se obtuvieron los siguientes resultados. (Tabla I)

Tabla I. Formación postgrado de los encuestados

\begin{tabular}{lrr}
\hline \multicolumn{1}{c}{$\begin{array}{c}\text { Formación } \\
\text { Post-Grado }\end{array}$} & Frecuencia & $\%$ \\
Doctorado & 1 & 1,5 \\
Máster & 10 & 14,9 \\
Experto & 14 & 20,9 \\
Especialista & 7 & 10,4 \\
Sin formación & 35 & 52,2 \\
Total & 67 & 100,0 \\
& \\
\hline
\end{tabular}

- La distribución de las respuestas por unidades fue la siguiente: (Tabla II)

Tabla II. Distribución de respuestas a la encuesta por unidades

\begin{tabular}{lrr}
\hline \multicolumn{1}{c}{ Unidad } & Frecuencia & $\%$ \\
Hospitalización & 9 & 13,4 \\
Médica. & & \\
Hospitalización & 2 & 3,0 \\
Quirúrgica. & & \\
Consultas & 1 & 1,5 \\
Urgencias. & 4 & 6,0 \\
UCl/REA. Adultos & 13 & 19,4 \\
Quirófano. & 4 & 6,0 \\
Hospital de día. & 5 & 7,5 \\
Pediatría & 18 & 26,9 \\
Neonatología. & & \\
Gestión. & 4 & 6,0 \\
Otros & 7 & 10,4 \\
Total & 67 & 100,0 \\
& & \\
\hline
\end{tabular}


- La distribución de las cargas familiares fue la que se muestra a continuación. (Tabla III).

Tabla III. Distribución de cargas familiares de la muestra.

\begin{tabular}{lrr}
\hline \multicolumn{1}{c}{ Cargas familiares } & Frecuencia & $\%$ \\
Vivo solo/a. & 6 & 9,0 \\
Vivo con pareja sin hijos. & 16 & 23,9 \\
Vivo con pareja e hijos. & 41 & 61,2 \\
Vivo con familia. & 2 & 3,0 \\
$\quad$ (no independizado). & & \\
Vivo con padres a mi cargo. & 1 & 1,5 \\
Otros. & 1 & 1,5 \\
Total & 67 & 100,0 \\
& & \\
\hline
\end{tabular}

- El 41,8\% de los encuestados había realizado algún trabajo de investigación en los últimos 2 años. (Tabla IV):

Tabla IV. Trabajos de investigación en los últimos dos años.

\begin{tabular}{lrr}
\hline $\begin{array}{l}\text { Trabajo de } \\
\text { investigación } \\
\text { en los últimos 2 } \\
\text { años }\end{array}$ & Frecuencia & $\%$ \\
$\mathrm{No}$ & & \\
$\mathrm{Si}$ & 39 & 58,2 \\
Total & 28 & 41,8 \\
\hline
\end{tabular}

- En cuanto a la frecuencia de lectura de artículos científicos, la distribución de la muestra fue la siguiente (Tabla $\mathrm{V}$ ):

Tabla V. Frecuencia de lectura científica

\begin{tabular}{lrr}
\hline $\begin{array}{l}\text { Frecuencia de lectura } \\
\text { científica }\end{array}$ & Frecuencia & $\%$ \\
La última semana. & 12 & 17,9 \\
Último mes. & 24 & 35,8 \\
Más de un mes. & 17 & 25,4 \\
Más de un año. & 11 & 16,4 \\
No lo recuerdo. & 3 & 4,5 \\
Total & 67 & 100 \\
\hline
\end{tabular}


- El 49,3\% de los encuestados había recibido formación relacionada con la investigación.

Las puntuaciones totales de la escala "THE BARRIERS" obtenidas de la encuesta se muestran a continuación (Tabla VI):

Tabla VI. Puntuaciones totales de la escala THE BARRIERS.

\begin{tabular}{|c|c|c|c|}
\hline Barreras & Media & Mediana & D.E. \\
\hline $\begin{array}{l}\text { [1. Los artículos/informes de investigación no están } \\
\text { disponibles.] }\end{array}$ & 2,63 & 3,00 & 894 \\
\hline $\begin{array}{l}\text { [2. Las implicaciones para la práctica no se presentan } \\
\text { con claridad.] }\end{array}$ & 2,94 & 3,00 & ,820 \\
\hline [3. Los análisis estadísticos no son comprensibles.] & 2,84 & 3,00 & ,863 \\
\hline $\begin{array}{l}\text { [4. La investigación no afecta a la práctica de la } \\
\text { enfermera.] }\end{array}$ & 2,14 & 2,00 & 1,094 \\
\hline [5. La enfermera desconoce las investigaciones.] & 3,33 & 4,00 & ,894 \\
\hline $\begin{array}{l}\text { [6. Las instalaciones no resultan adecuadas para su } \\
\text { implementación.] }\end{array}$ & 2,89 & 3,00 & ,914 \\
\hline $\begin{array}{l}\text { [7. La enfermería no tiene tiempo de leer la } \\
\text { investigación.] }\end{array}$ & 2,99 & 3,00 & 1,007 \\
\hline [8. La investigación no ha sido contrastada.] & 2,39 & 2,00 & ,831 \\
\hline $\begin{array}{l}\text { [9. La enfermería interpreta que las ventajas de aplicar } \\
\text { las nuevas ideas serán mínimas.] }\end{array}$ & 2,52 & 2,00 & ,827 \\
\hline $\begin{array}{l}\text { [10. La enfermería tiene dudas a la hora de aceptar los } \\
\text { resultados de la investigación.] }\end{array}$ & 2,70 & 3,00 & ,928 \\
\hline [11. La investigación tiene deficiencias metodológicas.] & 2,61 & 3,00 & ,743 \\
\hline $\begin{array}{l}\text { [12. La literatura correspondiente no está recogida en } \\
\text { un solo lugar.] }\end{array}$ & 3,18 & 3,00 & ,866 \\
\hline $\begin{array}{l}\text { [13. La enfermería no se siente con suficiente autoridad } \\
\text { como para cambiar los cuidados al paciente.] }\end{array}$ & 3,18 & 3,00 & ,815 \\
\hline $\begin{array}{l}\text { [14. La enfermería cree que los resultados no son } \\
\text { generalizables a su entorno.] }\end{array}$ & 2,62 & 3,00 & 823 \\
\hline $\begin{array}{l}\text { [15. La enfermería está aislada respecto a otros } \\
\text { profesionales con los que comentar los resultados de la } \\
\text { investigación.] }\end{array}$ & 3,05 & 3,00 & ,867 \\
\hline $\begin{array}{l}\text { [16. La enfermería ve en la investigación pocos } \\
\text { beneficios para su actividad profesional.] }\end{array}$ & 2,67 & 2,50 & 1,057 \\
\hline $\begin{array}{l}\text { [17. Los artículos/informes de investigación se publican } \\
\text { con mucha demora.] }\end{array}$ & 2,81 & 3,00 & ,856 \\
\hline [18. Los médicos no colaboran en la implementación.] & 3,26 & 4,00 & ,867 \\
\hline [19. Los directivos no permiten su implementación.] & 2,83 & 3,00 & ,874 \\
\hline $\begin{array}{l}\text { [20. La enfermería no percibe como relevantes los } \\
\text { resultados de la investigación para su aplicación en la } \\
\text { práctica.] }\end{array}$ & 2,74 & 3,00 & ,997 \\
\hline $\begin{array}{l}\text { [21. No existe una necesidad documentada para un } \\
\text { cambio de práctica.] }\end{array}$ & 2,58 & 3,00 & ,882 \\
\hline $\begin{array}{l}\text { [22. Las conclusiones planteadas no están } \\
\text { debidamente justificadas.] }\end{array}$ & 2,39 & 2,00 & ,797 \\
\hline [23. La literatura divulga resultados contradictorios.] & 2,42 & 2,00 &, 770 \\
\hline $\begin{array}{l}\text { [24. La investigación no está redactada con claridad, ni } \\
\text { invita a su lectura.] }\end{array}$ & 2,79 & 3,00 & ,786 \\
\hline [25. El resto de personal de enfermería no apoya su & 2,80 & 3,00 & ,833 \\
\hline
\end{tabular}


implementación.]

[26. La enfermería está poco dispuesta a $2,96 \quad 3,00 \quad$,960 cambiar/probar las nuevas ideas planteadas.]

[27. La cantidad de información de investigación es $2,58 \quad 2,50 \quad, 860$ aplastante.]

$\begin{array}{lllll}\text { [28. La enfermería no se siente capaz de evaluar la } & 2,92 & 3,00 & , 933\end{array}$

calidad de la investigación.]

[29. No hay tiempo suficiente en el trabajo para $3,16 \quad 3,00 \quad 931$

implementar nuevas ideas.]

\section{FACTORES}

Profesional

Organización

$22,47 \quad 23,0000 \quad 5,1267$

Calidad Investigación

$22,94 \quad 23,0000 \quad 4,6085$

Presentación accesibilidad

Las barreras identificadas con mayor puntuación fueron:

1. "La enfermera desconoce las investigaciones" media=3.33 $(\mathrm{DE}=0.89)$;

2. "Los médicos no colaboran en la implementación" media=3.26 ( $\mathrm{DE}=0.86)$;

3. "La literatura correspondiente no está recogida en un solo lugar" media=3.18 $(\mathrm{DE}=0.86)$ y

4. "La enfermería no se siente con suficiente autoridad como para cambiar los cuidados al paciente" media=3.18 $(\mathrm{DE}=0.81)$.

La suma de las puntuaciones máximas de la escala arroja un valor de 116 puntos siendo la media de las sumas totales de los profesionales encuestados de 74,95. Mediana 74 y DE: 13,57. Rango: 45-98.

Teniendo en cuenta las demás variables estudiadas, se determinó si existían o no diferencias en la puntuación de la escala en función de dichas variables, Las cuales se decriben a continuación. (Tabla VII)

Tabla VII. Resumen de las puntuaciones totales medias de la escala "THE BARRIERS" en función de las distintas variables estudiadas.

\begin{tabular}{|c|c|c|c|c|c|}
\hline Variable & Grupos & $\mathrm{n}$ & $\begin{array}{c}\text { Suma escala THE } \\
\text { BARRIERS } \\
\text { (Media) }\end{array}$ & $\begin{array}{l}\text { Diferencia } \\
\text { de Medias }\end{array}$ & Sig. \\
\hline \multirow[t]{2}{*}{ Edad } & $<40$ & 33 & 74.33 & \multirow[t]{2}{*}{5.93} & \multirow[t]{2}{*}{0.08} \\
\hline & $>=40$ & 34 & 80.26 & & \\
\hline \multirow[t]{2}{*}{ Sexo } & Hombre & 11 & 80.91 & \multirow[t]{2}{*}{4.26} & \multirow[t]{2}{*}{0.35} \\
\hline & Mujer & 56 & 76.64 & & \\
\hline \multirow{2}{*}{$\begin{array}{c}\text { Experiencia } \\
\text { como enfermera. }\end{array}$} & $<=18$ & 34 & 74.47 & \multirow[t]{2}{*}{5.832} & \multirow[t]{2}{*}{0.08} \\
\hline & $>18$ & 33 & 80.30 & & \\
\hline \multirow{2}{*}{$\begin{array}{c}\text { Formación Post- } \\
\text { Grado }\end{array}$} & $\mathrm{Si}$ & 32 & 81.84 & \multirow[t]{2}{*}{8.615} & \multirow[t]{2}{*}{0.01} \\
\hline & No & 35 & 73.23 & & \\
\hline \multirow{2}{*}{$\begin{array}{c}\text { Trabajo } \\
\text { Investigación } 2 \\
\text { años }\end{array}$} & $\mathrm{Si}$ & 28 & 81.75 & \multirow[t]{2}{*}{7.571} & \multirow[t]{2}{*}{0.02} \\
\hline & No & 39 & 74.18 & & \\
\hline \multirow{2}{*}{$\begin{array}{l}\text { Formación } \\
\text { investiqación }\end{array}$} & $\mathrm{Si}$ & 33 & 77.33 & \multirow[t]{2}{*}{0.02} & \multirow[t]{2}{*}{0.99} \\
\hline & No & 34 & 77.35 & & \\
\hline
\end{tabular}




\begin{tabular}{|c|c|c|c|c|c|}
\hline \multirow[t]{3}{*}{ Cargas familiares } & $\mathrm{Si}$ & 45 & 78.47 & \multirow[t]{2}{*}{3.421} & \multirow[t]{2}{*}{0.34} \\
\hline & No & 22 & 75.05 & & \\
\hline & \multicolumn{3}{|c|}{$\begin{array}{l}\text { Experiencia como enfermera } \\
\text { /Puntuación escala The Barriers. }\end{array}$} & $\begin{array}{l}\text { Correlación } \\
\text { Pearson }\end{array}$ & Sig. \\
\hline
\end{tabular}

- Resultados de las cuestiones de respuesta libre.

Por otro lado, la escala THE BARRIERS de S.Funk proporciona tres ítems más de valoración libre, a saber:

1. ¿Hay otras circunstancias que usted piensa que son barreras para la utilización de la investigación? Si es así, por favor enumere y califique cada una en la escala: (1) Nunca, (2) En menor grado, (3) En grado moderado, (4) En gran parte. (Tabla VIII):

Tabla VIII. Resumen de las respuestas de texto libre a la pregunta "¿Hay otras circunstancias que usted piensa que son barreras para la utilización de la investigación?"

\begin{tabular}{l|c}
\hline Barrera & Frecuencia \\
\hline Apoyo metodológico insuficiente & 1 \\
\hline Dificultad para acudir a congresos & 1 \\
\hline Falta de apoyo institucional & 2 \\
\hline Falta de formación & 6 \\
\hline Falta de implicación e interés & 2 \\
\hline Falta de medios & 1 \\
\hline Falta de motivación & 3 \\
\hline Falta de reconocimiento de carrera profesional & 1 \\
\hline Falta de tiempo & 8 \\
\hline Falta de trabajo en equipo & 1 \\
\hline Idioma & 1 \\
\hline Incompatibilidad de función investigadora con asistencial & 1 \\
\hline Necesidad de consenso & 1 \\
\hline Recursos informáticos inadecuados & 1 \\
\hline
\end{tabular}

Podemos observar cómo la falta de tiempo es la barrera que más se repite, seguida de la falta de formación.

2. Ante la pregunta "¿Cuál de todos los elementos anteriores cree que son los TRES mayores obstáculos para el uso de la investigación?"

Las tres barreras más nombradas como tales fueron la "falta de tiempo" $\left(\mathrm{n}^{0} 29\right)$, la "falta de autoridad de la enfermería" ( $\left.\mathrm{n}^{\circ} 13\right)$ y "los médicos no colaboran en la implementación" (nำ18).

3. ¿Cuáles son las circunstancias que usted piensa que facilitan la utilización de la investigación?

De los facilitadores que indican los encuestados los que se repiten con más frecuencia son los relativos al tiempo reconocido dentro de la jornada laboral $(n=8)$ y la 
formación en investigación $(n=8)$, seguidos de los relativos a la motivación del personal en investigación por parte de los responsables de enfermería $(n=7)$.

\section{DISCUSIÓN}

Aunque la falta de tiempo se identifica como la principal barrera para utilizar la investigación $^{(7)}$, en este estudio aparece por detrás de otras como el desconocimiento de las investigaciones, la falta de colaboración, o que la información no está recogida en un solo lugar. Posiblemente esto se debe a que una gran parte de los profesionales que han contestado el cuestionario pertenecen a unidades con más posibilidad de cumplimentar la encuesta. Sin embargo el tiempo aparece como una de las principales barreras en las respuestas de texto libre.

Por grupos de edad, observamos una mayor puntuación de la escala THE BARRIERS en mayores de 40 años, aunque esta diferencia no es estadísticamente significativa $(p=0.08)$, podemos sospechar que se debe a haber usado para el estudio una muestra escasa, ya que sí existan diferencias en las barreras percibidas en función de la experiencia como enfermera $(p=0.03)$, ya que la experiencia laboral es un factor asociado a la edad.

Encontramos diferencias significativas en función de no tener o tener formación postgrado, pero es este grupo de profesionales con mayor puntuación de media en la escala THE BARRIERS. Éste fenómeno ocurre también en el grupo de profesionales que han realizado algún trabajo de investigación en los últimos 2 años.

En comparación con los resultados obtenidos a nivel estatal en el estudio de MorenoCasbas et $\mathrm{al}^{(7)}$. se encuentran diferencias en cuanto a las puntuaciones medias de cada factor (Tabla IX).

Tabla IX. Resumen de las puntuaciones medias de la escala "THE BARRIERS" desglosadas por factores. Comparación con el estucio a nivel nacional de Moreno-Casbas.

\begin{tabular}{lcccc}
\hline Factores & $\begin{array}{c}\text { Media } \\
\text { HUFA } \\
\end{array}$ & D.E. & $\begin{array}{c}\text { Media } \\
\text { España }\end{array}$ & D.E. \\
& 2014 & & 2010 & \\
Características del profesional & 22.47 & 5.12 & 20.21 & 5.45 \\
Características de la organización & 22.94 & 4.61 & 21.30 & 5.41 \\
Cualidades de la investigación & 13.62 & 3.91 & 11.99 & 4.75 \\
Presentación y accesibilidad de la & 15.91 & 3.43 & 14.55 & 3.83 \\
investigación & & & & \\
\hline
\end{tabular}

Como podemos ver, las enfermeras del HUFA encuentran mayores barreras para la utilización de la investigación.

Las barreras más puntuadas en el estudio a nivel nacional son "No hay tiempo suficiente en el trabajo para implementar nuevas ideas" con una media de 3.13 y DE de 1.08 y "La enfermería desconoce las investigaciones" media de 2.84 y DE de 1.14. Al compararlo con nuestro estudio, encontramos que la falta de tiempo aparece como la quinta barrera en cuanto a puntuación, sin embargo, la media obtenida por este ítem en este estudio es superior a la obtenida en el estudio de Moreno-Casbas et al. (3.16 frente a un 3.13). (Tablas X y XI). 
Tabla X. Principales barreras identificadas y su puntuación media en el estudio de Moreno-Casbas et al 2010.

\begin{tabular}{lll}
\hline Barreras. España 2010. Moreno-Casbas & Media & D.E. \\
No hay tiempo suficiente en el trabajo para implementar nuevas & & \\
ideas. & 3.13 & 1.08 \\
La enfermera desconoce las investigaciones & 2.84 & 1.14 \\
Los médicos no colaboran en la implementación & 2.79 & 1.31 \\
La enfermería está aislada respecto a otros profesionales con & 2.72 & 1.19 \\
los que comentar los resultados de la investigación & & \\
$\begin{array}{l}\text { Las instalaciones no resultan adecuadas para su } \\
\text { implementación }\end{array}$ & 2.69 & 1.28 \\
\hline
\end{tabular}

Tabla XI. Principales barreras identificadas y su puntuación media en nuestro estudio. 2014.

\begin{tabular}{lll}
\hline Barreras. HUFA 2014 & Media & D.E. \\
La enfermera desconoce las investigaciones & 3.33 & 0.89 \\
Los médicos no colaboran en la implementación & 3.26 & 0.86 \\
La literatura correspondiente no está recogida en un solo lugar & 3.18 & 0.86 \\
La enfermería no se siente con suficiente autoridad como para & 3.18 & 0.81 \\
$\begin{array}{l}\text { cambiar los cuidados al paciente } \\
\text { No hay tiempo suficiente en el trabajo para implementar nuevas } \\
\text { ideas. }\end{array}$ & $\mathbf{3 . 1 6}$ & 0.93 \\
\hline
\end{tabular}

\section{Limitaciones del estudio}

En primer lugar nos encontramos con un escaso porcentaje de respuesta al cuestionario probablemente influida por la capacidad de manejo de herramientas informáticas de cada profesional. Sin embargo, la posibilidad de envío vía e-mail permitió una rápida obtención de los datos y la posibilidad de llegar a un mayor número de profesionales.

Probablemente el porcentaje de respuesta es tan escaso debido a que los profesionales recibieron la encuesta a través del correo electrónico corporativo. Y en la gran mayoría de los casos tenían que contestarla en horario de trabajo. Paradójicamente, la falta de tiempo que las enfermeras aducen como la principal barrera para la utilización de la investigación es la que puede haber impedido en muchos casos que se responda la encuesta para evaluar dichas barreras.

Esta baja tasa de respuesta explica que la distribución de los profesionales encuestados por unidades, sea distinta a la distribución real de las enfermeras, obteniendo un alto porcentaje de respuestas proveniente de las unidades de Pediatría-Neonatología y Unidad de Cuidados Críticos.

\section{CONCLUSIONES}

Este estudio nos aporta una visión de las limitaciones que las enfermeras del HUFA tienen para investigar y utilizar esa investigación. La falta de tiempo, colaboración o tiempo siguen siendo obstáculos que de manera recurrente la enfermería debe ir eliminando. 
Una vez obtenidos los resultados se pretende orientar la actuación de la unidad de investigación y las necesidades de formación del personal en temas de investigación.

\section{REFERENCIAS}

1. García-Fernández FP. [Nursing research: an invisible reality?]. Enfermería clínica [Internet]. 2012 Jan [cited 2015 Dec 28];22(6):283-5. Available from: http://www.sciencedirect.com/science/article/pii/S1130862112001477

2. BOE.es - Documento BOE-A-1977-20006 [Internet]. [cited 2016 Feb 8]. Available from: https://www.boe.es/diario boe/txt.php?id=BOE-A-1977-20006

3. García-García I, Gozalbes Cravioto E. Investigación en Enfermería y en Historia de la Enfermería en España.Index de Enfermería. 2012;21(1-2):1004.

http://scielo.isciii.es/scielo.php?script=sci arttext\&pid=S1132$12962012000100023 \& \operatorname{lng}=e s \& n r m=i s o \& t \operatorname{lng}=e s$

3. Funk SG, Champagne MT, Wiese RA, Tornquist EM. BARRIERS: the barriers to research utilization scale. Appl Nurs Res. United States; 1991;4(1):39-45. http://barriers.web.unc.edu/

4. Funk SG, Tornquist EM, Champagne MT. Barriers and facilitators of research utilization. An integrative review. Nurs Clin North Am. School of Nursing, University of North Carolina at Chapel Hill, USA.; 1995;30(3):395-407.

5. Hunt JM. Barriers to research utilization. J Adv Nurs. England; 1996;23(3):423-5.

6. Moreno-Casbas T, Fuentelsaz-Gallego C, Gonzalez-Maria E, Gil de Miguel A. Barreras para la utilización de la investigación. Estudio descriptivo en profesionales de enfermería de la práctica clínica y en investigadores activos. Enferm Clin $2010 \quad$ May-Jun;20(3):153-164. http://www.sciencedirect.com/science/article/pii/S113086211000015X

Recibido: 19 de diciembre 2014; Aceptado: 2 de febrero 2016

ISSN 1695-6141

๑) COPYRIGHT Servicio de Publicaciones - Universidad de Murcia 